\title{
Overnight Changes in Lung Function of Obese Patients with Obstructive Sleep Apnoea
}

\author{
Laszlo Kunos $^{1} \cdot$ Zsofia Lazar $^{1} \cdot$ Fruzsina Martinovszky $^{1} \cdot$ Adam D. Tarnoki $^{2}$ • \\ David L. Tarnoki ${ }^{2}$ - Daniel Kovacs ${ }^{2} \cdot$ Bianka Forgo $^{2} \cdot$ Peter Horvath $^{1}$. \\ Gyorgy Losonczy $^{1} \cdot$ Andras Bikov $^{1}$
}

Received: 8 May 2016/ Accepted: 13 October 2016

(c) Springer Science+Business Media New York 2016

\begin{abstract}
Purpose Obstructive sleep apnoea (OSA) is a prevalent disorder, characterised by collapse of the upper airways during sleep. The impact of sleep-disordered breathing on pulmonary function indices is however currently not well described. The aim of the study was to evaluate diurnal change in lung function indices in a cohort of patients with OSA and relate pulmonary function changes to disease severity.

Methods 42 patients with OSA and 73 healthy control subjects participated in the study. Asthma and COPD were excluded in all volunteers following a clinical and spirometric assessment. Spirometry was then performed in all subjects in the evening and the morning following a polysomnography study.

Results There was no difference in evening or morning $\mathrm{FEV}_{1}$ or FVC between patients and control subjects $(p>0.05)$. Neither $\mathrm{FEV}_{1}$ nor FVC changed in control subjects overnight $(p>0.05)$. In contrast, $\mathrm{FEV}_{1}$ significantly increased from evening (2.18/1.54-4.46/L) to morning measurement (2.26/1.42-4.63/L) in OSA without any change in FVC. The FEV ${ }_{1}$ increase in OSA was related to male gender, obesity and the lack of treatment with statins or $\beta$-blockers (all $p<0.05$ ). A tendency for a direct correlation was apparent between overnight $\mathrm{FEV}_{1}$ change and RDI $(p=0.05, r=0.30)$.
\end{abstract}

Andras Bikov

andras.bikov@gmail.com

1 Department of Pulmonology, Semmelweis University, 1/C Dios arok, Budapest 1125, Hungary

2 Department of Radiology and Oncotherapy, Semmelweis University, 78a Ulloi ut, Budapest 1082, Hungary
Conclusions Diurnal variations in spirometric indices occur in patients with OSA and $\mathrm{FEV}_{1}$ appears to increase in subjects with OSA overnight. These changes occur in the absence of change in FVC and are directly related to the severity of OSA. These findings dictate a need to consider time of lung function measurement.

Keywords Forced expiratory volume in $1 \mathrm{~s} \cdot$ Lung function - Obstructive sleep apnoea $\cdot$ Overnight changes

\section{Introduction}

Obstructive sleep apnoea (OSA) is a common disorder which is characterised by intermittent complete or partial collapse of the upper airways during sleep. This results in overnight hypoxaemia and frequent arousals which may lead to excessive daytime sleepiness and the development or worsening of metabolic, cardiovascular and cognitive disorders [1].

OSA is a disorder which not only affects the upper airways but can also impact on the intra-thoracic airways. Indeed, reduced lung volumes, decreased elasticity and increased resistance of the lower airways are associated with more severe disease [2-5]. In addition, chronic airway diseases, including asthma and chronic obstructive pulmonary disease (COPD), influence the course of OSA $[6,7]$. Lower airway inflammation and oxidative stress are accelerated in OSA with a direct relationship between disease severity and the magnitude of lower airways inflammation [8]. It appears likely that OSA may also impact on lung volume mechanics.

To date, however there have been no studies evaluating overnight alterations in lung function indices in OSA. Theoretically, lung function may vary due to two 
main reasons. On one hand, overnight changes in airway broncho-reactive mediator concentrations were reported and may influence operational lung volumes [8-12]. On the other hand, OSA is associated with increased sympathetic and decreased parasympathetic tone during sleep, especially during apnoeic periods [13-15], which may result in alterations in resting bronchial tone [16-18].

The aim of the study was therefore to evaluate diurnal change in lung function indices in a cohort of patients with OSA and compare these with a healthy control cohort. Apart from providing improved understanding regarding pathophysiology in OSA, this evaluation has important clinical implications, namely when to perform lung function testing in patients with OSA.

\section{Methods}

\section{Study Subjects and Design}

115 adult volunteers were recruited (47 men, $51 \pm$ 15 years, BMI $27.1 \pm 5.8 \mathrm{~kg} / \mathrm{m}^{2}$ ). COPD and asthma were excluded by spirometry and rigorous assessment of medical history in all subjects. More specifically, we excluded patients if any of the evening or morning $\mathrm{FEV}_{1} / \mathrm{FVC}$ ratio was below 0.70 , or if they reported chronic or intermittent symptoms of wheezing, shortness of breath, chest tightness and cough. However, no bronchial provocation or bronchial reversibility test has been performed. Subjects who could not perform reliable and reproducible lung function tests were excluded. Subjects were recruited from patients attending Sleep Laboratory of Semmelweis University, Department of Pulmonology $(N=32)$, and through internet advertisement $(N=83)$, and none of them has previously been diagnosed with sleep-disordered breathing. Accordingly, no subject had used continuous positive airway pressure (CPAP) device prior to recruitment. Thirteen patients were current or ex-smokers, 42 subjects were previously diagnosed with hypertension, 15 had known diabetes, 28 had dyslipidaemia and 26 had allergic rhinitis. None of the subjects had respiratory tract infection 1 month prior to the study.

Medical history was taken, patients filled in the Epworth Sleepiness Scale, blood pressure and heart rate were measured and lung function tests were performed in the evening (between 7 and $8 \mathrm{pm}$ ). Subjects then attended a full-night polysomnography. Blood pressure and heart rate were measured and lung function tests were repeated between 7 and 8 am, within an hour after awakening. The study was approved by the local ethics committee (Semmelweis University TUKEB 30/2014) and volunteers gave their written informed consent.

\section{Polysomnography}

Polysomnography was performed as described previously [9] using Somnoscreen Plus Tele PSG (Somnomedics GmbH, Germany) according to the guidelines [19]. Apnoea-hypopnoea index (AHI), respiratory disturbance index (RDI) and oxygen desaturation index (ODI) were recorded and used as indices for OSA severity.

\section{Spirometry and Blood Pressure Measurement}

Spirometry was performed with the Otthon device (Thor Medical Systems, Budapest, Hungary) according to the European Respiratory Society guidelines [20]. At least three technically acceptable lung function measurements were performed (the difference between the two highest values for forced vital capacity (FVC) or forced expiratory volume in one second $\left(\mathrm{FEV}_{1}\right)$ was $\left.<0.150 \mathrm{~L}\right)$. The highest values of $\mathrm{FEV}_{1}$ and $\mathrm{FVC}$ were recorded. Blood pressure was taken using a mercury sphygmomanometer with cuff placed on the left upper arm.

\section{Statistical Analysis}

GraphPad Prism 5.0 (GraphPad Software, San Diego, CA, US) and Statistica 12 (StatSoft, Inc., Tulsa, OK, US) were used for statistical analyses. Data distribution was assessed with Kolmogorov-Smirnov test which showed a nonparametric distribution for $\mathrm{FEV}_{1}$ and $\mathrm{FVC}$, but parametric distribution for $\mathrm{FEV}_{1} / \mathrm{FVC}$. Unpaired $t$ test, Mann-Whitney and $\chi^{2}$ tests were used to compare clinical variables between OSA and control groups. Evening-to-morning changes in lung function were evaluated with Wilcoxon test and were expressed as $\Delta=$ morning - evening value. The relationships between lung function values and clinical variables were assessed with Spearman's test. Multiple logistic regression was applied to analyse the effect of potential covariates for the relationship between lung function indices and OSA severity. A $p$ value $<0.05$ was considered significant. Data are expressed as mean \pm standard deviation and median/range/for parametric and non-parametric data, respectively.

\section{Results}

\section{Subject Demographics and Comparison of OSA and Control Subjects}

Forty-two subjects were diagnosed with OSA (AHI $\geq 5 / \mathrm{h})$ and 73 volunteers were considered as controls $(\mathrm{AHI}<5 / \mathrm{h})$. Compared to control subjects, patients with OSA were more frequently male, older and had a greater BMI. 
Patients also had an increased rate of comorbidities including hypertension and dyslipidaemia $(p<0.05)$. Twenty-three patients with OSA and thirteen controls were obese $\left(B M I \geq 30 \mathrm{~kg} / \mathrm{m}^{2}\right)$. The proportion of restrictive lung disease (FVC as well as $\mathrm{FEV}_{1}<80 \%$ pred. and $\mathrm{FEV}_{1} / \mathrm{FVC}>0.70$ ) was similar in obese patients with OSA $(61 \%)$ and obese controls $(46 \%, p=0.39)$. A higher number of patients with OSA were treated with statins, $\mathrm{Ca}$ channel blockers, angiotensin-converting enzyme inhibitors (ACEI) and angiotensin receptor blockers (ARB, $p<0.01)$; however, no difference was found in the frequency of $\beta$-blocker users $(p=0.72$; Table 1$)$. There was no difference in $\mathrm{FEV}_{1}, \mathrm{FVC}$ or $\mathrm{FEV}_{1} / \mathrm{FVC}$ between the two groups either when they were measured in the evening or in the morning (all $p>0.05$; Table 2).

\section{Relationship Between Spirometric Indices and Disease Severity in Patients with OSA}

There was an indirect relationship between $\mathrm{FEV}_{1}$ measured in the evening (expressed as $\%$ predicted) and AHI ( $p=0.02, r=-0.34)$, ODI $(p=0.02, r=-0.35)$ as well as RDI $(p=0.01, r=-0.39)$. Similarly, evening FVC ( $\%$ predicted) was associated with AHI $(p=0.04$, $r=-0.31$; Fig. 1$)$, ODI $(p=0.04, r=-0.31)$ and RDI $(p<0.01, r=-0.39) . \mathrm{FEV}_{1}$ in the morning (\% predicted)
Table 2 Lung function values in the evening and morning

\begin{tabular}{clll}
\hline & OSA & Control & $p$ \\
\hline FEV $_{1}(\mathrm{~L})$ & & & \\
Evening & $2.18 / 1.54-4.46$ & $2.75 / 1.45-5.81$ & 0.12 \\
Morning & $2.26 / 1.42-4.63$ & $2.77 / 1.52-5.61$ & 0.19 \\
$p$ & 0.02 & 0.74 & \\
FVC (L) & & & \\
Evening & $2.60 / 1.83-5.32$ & $3.28 / 1.79-6.51$ & 0.17 \\
Morning & $2.71 / 1.77-5.64$ & $3.17 / 1.79-5.91$ & 0.23 \\
$p$ & 0.19 & 0.97 & \\
FEV $/$ FVC & & & 0.26 \\
Evening & $0.84 \pm 0.05$ & $0.85 \pm 0.07$ & 0.11 \\
Morning & $0.84 \pm 0.06$ & $0.86 \pm 0.07$ & \\
$p$ & 0.78 & 0.54 & \\
\hline
\end{tabular}

Forced expiratory volume in $1 \mathrm{~s}\left(\mathrm{FEV}_{1}\right)$, forced vital capacity $(\mathrm{FVC})$ values and $\mathrm{FEV}_{1} / \mathrm{FVC}$ ratios in the evening and morning in patients with obstructive sleep apnoea (OSA) and control subjects. Data are expressed as median/range/for $\mathrm{FEV}_{1}$ and $\mathrm{FVC}$ and as mean \pm standard deviation for $\mathrm{FEV}_{1} / \mathrm{FVC}$

was inversely related to RDI ( $p=0.03, r=-0.33$ ), and morning FVC values (\% predicted) were negatively associated with AHI $(p=0.03, r=-0.33)$ and RDI $(p=0.01, r=-0.38)$. Neither $\mathrm{FEV}_{1} / \mathrm{FVC}$ ratio nor absolute lung volumes were related to OSA parameters. In
Table 1 Comparison of clinical variables of study participants

\begin{tabular}{lllr}
\hline & OSA & Control & \multicolumn{1}{c}{$p$} \\
\hline Age (years) & $64 / 36-74$ & $46 / 20-74$ & $<0.01$ \\
Gender (male/female) & $23 / 19$ & $24 / 49$ & 0.02 \\
BMI $\left(\mathrm{kg} / \mathrm{m}^{2}\right)$ & $31.1 \pm 6.3$ & $24.8 \pm 4.0$ & $<0.01$ \\
Hypertension $(n)$ & $25 / 17$ & $17 / 56$ & $<0.01$ \\
Diabetes $(n)$ & $8 / 34$ & $7 / 66$ & 0.14 \\
Dyslipidaemia $(n)$ & $19 / 23$ & $9 / 64$ & $<0.01$ \\
Allergic rhinitis $(n)$ & $6 / 36$ & $20 / 53$ & 0.09 \\
Medication usage (yes/no) & & & \\
Statins & $11 / 31$ & $1 / 72$ & $<0.01$ \\
Ca channel blockers & $8 / 34$ & $2 / 71$ & $<0.01$ \\
ACEI or ARB & $14 / 28$ & $7 / 66$ & $<0.01$ \\
B-blockers & $8 / 34$ & $12 / 61$ & 0.72 \\
Smoking history (ever/never smoker) & $5 / 37$ & $8 / 65$ & 0.87 \\
Cigarette pack years & $0 / 0-30$ & $0 / 0-33$ & 0.16 \\
Epworth sleepiness scale & $5 / 0-14$ & $6 / 0-14$ & 0.10 \\
AHI (1/h) & $17.0 / 5.2-93.3$ & $0.9 / 0.0-4.8$ & $<0.01$ \\
ODI (1/h) & $18.8 / 0.0-119.4$ & $1.0 / 0.0-18.3$ & $<0.01$ \\
RDI (1/h) & $27.0 / 14.2-103.5$ & $10.5 / 0.6-24.2$ & $<0.01$ \\
\hline Significant differences & & & \\
\hline
\end{tabular}

Significant differences were observed in age, gender, body mass index (BMI), prevalence of hypertension, dyslipidaemia, statin, Ca channel blocker as well as angiotensin-converting enzyme inhibitor (ACEI) or angiotensin receptor blocker (ARB) treatment, apnoea-hypopnoea index (AHI), oxygen desaturation index (ODI) and respiratory disturbance index (RDI). Data are expressed as mean \pm standard deviation for parametric or median/range/for non-parametric data 


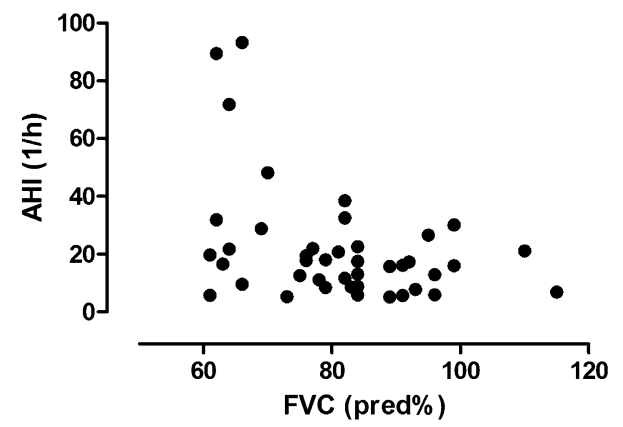

Fig. 1 Relationship between forced vital capacity and apnoeahypopnoea index in OSA. There was a significant relationship between apnoea-hypopnoea index (AHI) and forced vital capacity (FVC) in OSA measured in the evening ( $p=0.04, r=-0.31)$

addition, no correlation was observed between lung function and Epworth Sleepiness Scale $(p>0.05)$.

To assess the effect of potential covariates (age, gender, obesity, comorbidities, medication usage and smoking) on the relationship between lung function and OSA severity, the OSA group was divided into mild (AHI 5-14.9 1/h, $n=17$ ), moderate (AHI 15-29.9 1/h, $n=17$ ) and severe (AHI $>301 / \mathrm{h}, \quad n=8$ ) subgroups. Multiple logistic regression revealed BMI, hypertension, diabetes, dyslipidaemia as well as treatment with statin, ACEI, ARB and $\beta$-blocker as significant covariates (all $p<0.05$ ). In contrast, age, gender, smoking, allergy and therapy with a $\mathrm{Ca}$ channel blocker did not contribute to the relationship (all $p>0.05)$.

\section{Overnight Changes in Spirometric Indices}

In OSA, there was a significant increase in $\mathrm{FEV}_{1}$ from the evening to the morning $(p=0.02)$, while no difference in FVC $(p=0.19)$ or $\mathrm{FEV}_{1} / \mathrm{FVC}(p=0.78)$ was noted. Morning and evening $\operatorname{FEV}_{1}(p=0.74), \operatorname{FVC}(p=0.97)$ or $\mathrm{FEV}_{1} / \mathrm{FVC}(p=0.54$; Table 2$)$ were not different in the control group. Analysing factors associated with lung function changes in OSA, significant relationships were observed between $\Delta \mathrm{FEV}_{1}$ and height ( $p=0.02, r=0.35$ ), smoking status $(p<0.01, r=-0.44)$ and cigarette pack years $(p<0.01, r=-0.44)$, and a tendency for a positive correlation with $\operatorname{RDI}(p=0.05, r=0.30 ;$ Fig. 2$)$ was also noted. No correlation was observed between changes in lung function and Epworth Sleepiness Scale $(p>0.05)$. With respect to the effect of gender, a significant $\mathrm{FEV}_{1}$ change was observed only in males $(p=0.03)$, but not in females $(p=0.25)$. In addition, $\mathrm{FEV}_{1}$ increased only in patients with OSA not using statins $(p=0.02)$ or $\beta$-blockers $(p=0.03)$, while there was no change in patients on treatment with statins $(p=0.97)$ or $\beta$-blockers $(p=0.54)$. Ca channel blocker, ACEI or ARB therapy did not affect the results. When the OSA group was divided into obese $(n=23)$ and

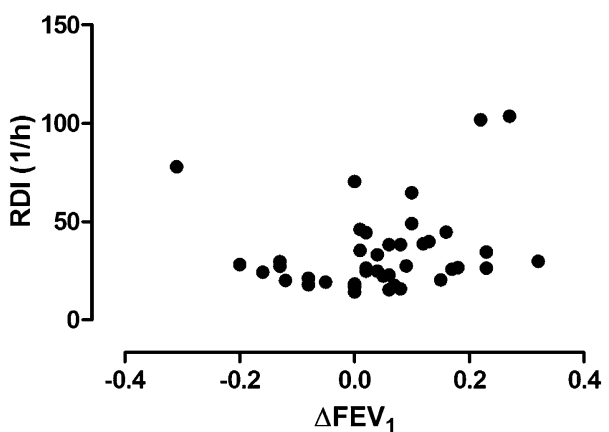

Fig. 2 Relationship between overnight $\mathrm{FEV}_{1}$ change and respiratory disturbance index in OSA. There was a tendency for a direct relationship between respiratory disturbance index (RDI) and overnight changes in forced expiratory volume in $1 \mathrm{~s}$ in OSA $(p=0.05$, $r=0.30$ )

non-obese subgroups $(n=19)$, a significant $\mathrm{FEV}_{1}$ increase was found only in obese patients $(p=0.03)$, while no difference was observed in non-obese subjects $(p=0.33)$. In contrast, there was no change either in the thirteen obese $(p=0.90)$ or non-obese $(p=0.63)$ controls.

\section{Overnight Changes in Blood Pressure and Heart Rate}

In OSA, there was no overnight change either in systolic (evening to morning: $140 / 100-190$ to $140 / 110-190 \mathrm{mmHg}$, $p=0.97)$ or diastolic $(80 / 70-120$ to $85 / 60-120 \mathrm{mmHg}$, $p=0.97$ ) blood pressure values. However, heart rate increased in the morning (74/56-91 to $79 / 60-94 / 1 / \mathrm{min}$, $p=0.01)$. In contrast, a significant drop in morning systolic $(125 / 90-170$ to $120 / 100-160 \mathrm{mmHg}, p<0.01)$ and diastolic $(80 / 65-110$ to $75 / 60-100 \mathrm{mmHg}, p<0.01)$ blood pressure was observed in control subjects with no change in heart rate (from 76/44-96 to 76/51-97/1/min, $p=0.24$ ). Neither absolute values nor changes in blood pressure or heart rate were related to lung function indices or their overnight changes (all $p>0.05$ ).

\section{Discussion}

OSA is a common disorder with an unclear pathophysiology. Despite the fact that the first report on the association between lung volumes and OSA was published already 30 years ago [3], changes in the behaviour of the intrathoracic airways have not been investigated in this disorder. Our study was the first to demonstrate diurnal change in select lung function parameters in a cohort of wellcharacterised patients with OSA with pathophysiological and clinical implications. We found a significant $80-\mathrm{mL}$ overnight increase in $\mathrm{FEV}_{1}$ in patients with OSA which directly related to the overnight apnoeic burden. 
A reduction in end-tidal lung volumes may lead to a heightened potential for collapse of the upper airways [5]. Indeed, the relationship between reduced lung volume, increased respiratory resistance and increased severity of OSA is already described [2-4]. In keeping with these studies, we also found a significant, albeit weak association between spirometric indices and OSA severity. Although obesity may lead to restrictive ventilatory defect, there is a growing body of evidence linking OSA to impaired lung function; necessitating the investigation of sleep-disordered breathing in patients with restrictive parenchymal lung diseases.

Interestingly, a significant inverse relationship was also observed between changes in lung function indices and smoking status. Smoking even without COPD is associated with impaired respiratory mechanics [21]. Our study suggests that smoking non-COPD OSA patients are prone to develop overnight fall in $\mathrm{FEV}_{1}$, a feature known in chronic respiratory diseases [22].

The reasons for the alterations in lung function observed in the current study remain to be determined. Changes in lung function potentially result from nocturnal release of broncho-reactive mediators. Overnight increase in the levels of potentially bronchoconstrictive 8-isoprostane [10] and bronchodilator nitric oxide [11, 12] has previously been reported in the exhaled breath samples in OSA subjects and these mediator changes may be responsible for the findings reported in the current study. Another possible explanation is the augmented adrenal-sympathetic tone during the night in OSA patients [13, 14, 23]. Although bronchi are innervated by parasympathetic rather than sympathetic nerves in mammals [16, 18], circulating adrenalin is a potent bronchodilator in humans [17]. During apnoeic periods, parasympathetic tone is decreased [24] and repeated arousal stimuli are associated with increased sympathetic activity [25], which may also lead to bronchodilation. This imbalance between sympathetic and parasympathetic tone may last even during wakefulness $[15,26]$. In the current study, this fact was supported by the lack of physiological blood pressure dip and the increase in heart rate. Although we did not measure circulating catecholamines, previous studies report an increase in the concentration of blood adrenaline overnight in OSA [23] and this may contribute to a propensity to bronchodilation [27].

Not surprisingly, we found significant differences in age, gender, BMI, the prevalence of comorbidities and medications between patients and controls. Age did not influence our results; however, $\mathrm{FEV}_{1}$ increased only in male patients. The precise explanation for our findings is not known, but there are certain gender differences in the distribution of adipose tissue, upper airway anatomy, control of ventilation and release of hormones [28]. We found that BMI was a covariate for the relationship of lung function and OSA severity. In addition, significant evening-to-morning increase in $\mathrm{FEV}_{1}$ was present only in obese patients with OSA. Obesity may lead to the development of airway hyper-responsiveness both in animals and humans [29]. The underlying mechanisms include both mechanical (increased abdominal and chest wall mass as well as shallow breathing) and humoral (circulating inflammatory cytokines and hormones, such as leptin or adiponectin) factors [29]. However, obesity did not exclusively explain our findings, as no change in lung function was observed in obese non-OSA subjects.

Patients with OSA were more frequently treated with medications potentially affecting airway patency. We found that statin and $\beta$-blocker usage significantly blunted evening-to-morning $\mathrm{FEV}_{1}$ elevation. It is known that chronic treatment with statins [30] and $\beta$-blockers [31] inhibits airway hyper-reactivity. Furthermore, the sympathetic blockade by $\beta$-blockers may contribute to the lack of $\mathrm{FEV}_{1}$ changes. Of note, although gender, obesity and drug treatment modified our findings, this study was not powered to analyse causality of these factors. Therefore, these effects need to be investigated in further studies.

The limitations of this study include the relatively low sample size and the lack of objective measurement of bronchial hyper-reactivity. The sample size was estimated to find differences in lung function between the OSA and control groups. Although we reported that there were significant differences when the groups were divided according to gender, obesity and medication usage, the low sample size in the subgroups limits our ability to fully determine the influence of these factors.

Lung function exhibits some degree of diurnal variation in health and chronic airway diseases [22, 32]. It is known that patients with chronic airway disease other than asthma (i.e. COPD or allergic rhinitis) and even healthy subjects may show some degree of bronchial hyper-responsiveness [33], and obesity may itself predispose towards hyper-responsiveness without other symptoms of asthma [29]. As symptoms are imperative constituents of asthma definition, we believe that by rigorous assessment of medical history, bronchial asthma was reliably excluded. However, we did not perform bronchial reversibility or provocation test. Therefore, further studies are needed to evaluate airway hyper-responsiveness in obese OSA. Furthermore, as variability of lung function is well known even in healthy subjects, multiple evening-to-morning assessment would provide stronger evidence for our findings. Finally, although $\mathrm{FEV}_{1}$ is more commonly used to assess airway obstruction than the measures of airway/respiratory resistance, independent studies using body plethysmography or forced oscillation technique would be highly informative in this context. 
In conclusion, $\mathrm{FEV}_{1}$ increased from evening to morning in patients with untreated OSA and the magnitude of this change was directly related to the severity of OSA. These findings may be explained by increased sympathetic tone or elevated levels of bronchodilator mediators caused by untreated sleep-disordered breathing. The significant increase in $\mathrm{FEV}_{1}$ value in some patients (i.e. those with more severe OSA) may need to be taken into account when interpreting results of lung function tests. Further studies are needed to evaluate the mechanisms underlying these changes and their implications in patients with OSA.

Acknowledgments The authors are grateful to Mrs. Monika Banlaky for her assistance in polysomnographic measurements and to Dr. James H Hull for English corrections. The authors are also grateful to Thor Medical and Elektro-Oxigén Inc. for providing spirometers and polysomnographic devices, respectively. Hungarian Respiratory Society provided financial support in the form of funding to David L Tarnoki (2014). This publication was supported by the Janos Bolyai Research Scholarship of the Hungarian Academy of Sciences to Andras Bikov.

\section{Compliance with Ethical Standards}

Conflict of interest The authors declare that they have no conflict of interest.

\section{References}

1. Strollo PJ Jr, Rogers RM (1996) Obstructive sleep apnea. N Engl J Med 334(2):99-104. doi:10.1056/NEJM199601113340207

2. Abdeyrim A, Zhang Y, Li N, Zhao M, Wang Y, Yao X, Keyoumu Y, Yin T (2015) Impact of obstructive sleep apnea on lung volumes and mechanical properties of the respiratory system in overweight and obese individuals. BMC Pulm Med 15:76. doi:10. 1186/s12890-015-0063-6

3. Onal E, Leech JA, Lopata M (1985) Relationship between pulmonary function and sleep-induced respiratory abnormalities. Chest 87(4):437-441

4. Abdeyrim A, Li N, Shao L, Heizhati M, Wang Y, Yao X, Abulikemu S, Zhang D, Chang G, Yin T, Li C, Meng J, Zhao M, Zhou L, Hong J, Zhang Y (2016) What can impulse oscillometry and pulmonary function testing tell us about obstructive sleep apnea: a case-control observational study? Sleep Breath 20(1):61-68. doi:10.1007/s11325-015-1185-z

5. Owens RL, Malhotra A, Eckert DJ, White DP, Jordan AS (2010) The influence of end-expiratory lung volume on measurements of pharyngeal collapsibility. J Appl Physiol 108(2):445-451. doi:10. 1152/japplphysiol.00755.2009

6. Bednarek M, Plywaczewski R, Jonczak L, Zielinski J (2005) There is no relationship between chronic obstructive pulmonary disease and obstructive sleep apnea syndrome: a population study. Respiration 72(2):142-149. doi:10.1159/000084044

7. Teodorescu M, Barnet JH, Hagen EW, Palta M, Young TB, Peppard PE (2015) Association between asthma and risk of developing obstructive sleep apnea. JAMA 313(2):156-164. doi:10.1001/jama.2014.17822

8. Bikov A, Hull JH, Kunos L (2016) Exhaled breath analysis, a simple tool to study the pathophysiology of obstructive sleep apnoea. Sleep Med Rev 27:1-8. doi:10.1016/j.smrv.2015.07. 005
9. Kunos L, Bikov A, Lazar Z, Korosi BZ, Benedek P, Losonczy G, Horvath I (2015) Evening and morning exhaled volatile compound patterns are different in obstructive sleep apnoea assessed with electronic nose. Sleep Breath 19(1):247-253. doi:10.1007/ s11325-014-1003-z

10. Carpagnano GE, Kharitonov SA, Resta O, Foschino-Barbaro MP, Gramiccioni E, Barnes PJ (2003) 8-Isoprostane, a marker of oxidative stress, is increased in exhaled breath condensate of patients with obstructive sleep apnea after night and is reduced by continuous positive airway pressure therapy. Chest 124(4):1386-1392

11. Chua AP, Aboussouan LS, Minai OA, Paschke K, Laskowski D, Dweik RA (2013) Long-term continuous positive airway pressure therapy normalizes high exhaled nitric oxide levels in obstructive sleep apnea. J Clin Sleep Med 9(6):529-535. doi:10.5664/jcsm. 2740

12. Olopade CO, Christon JA, Zakkar M, Hua C, Swedler WI, Scheff PA, Rubinstein I (1997) Exhaled pentane and nitric oxide levels in patients with obstructive sleep apnea. Chest 111(6):1500-1504

13. Narkiewicz K, van de Borne PJ, Montano N, Dyken ME, Phillips BG, Somers VK (1998) Contribution of tonic chemoreflex activation to sympathetic activity and blood pressure in patients with obstructive sleep apnea. Circulation 97(10):943-945

14. Marrone O, Riccobono L, Salvaggio A, Mirabella A, Bonanno A, Bonsignore MR (1993) Catecholamines and blood pressure in obstructive sleep apnea syndrome. Chest 103(3):722-727

15. Hakim F, Gozal D, Kheirandish-Gozal L (2012) Sympathetic and catecholaminergic alterations in sleep apnea with particular emphasis on children. Front Neurol 3:7. doi:10.3389/fneur.2012. 00007

16. Cabezas GA, Graf PD, Nadel JA (1971) Sympathetic versus parasympathetic nervous regulation of airways in dogs. J Appl Physiol 31(5):651-655

17. Larsson K (1985) Studies of sympatho-adrenal reactivity and adrenoceptor function in bronchial asthma. Eur $\mathbf{J}$ Respir Dis Suppl 141:1-52

18. Canning BJ (2006) Reflex regulation of airway smooth muscle tone. J Appl Physiol 101(3):971-985. doi:10.1152/japplphysiol. 00313.2006

19. Kushida CA, Littner MR, Morgenthaler T, Alessi CA, Bailey D, Coleman J Jr, Friedman L, Hirshkowitz M, Kapen S, Kramer M, Lee-Chiong T, Loube DL, Owens J, Pancer JP, Wise M (2005) Practice parameters for the indications for polysomnography and related procedures: an update for 2005. Sleep 28(4):499-521

20. Miller MR, Hankinson J, Brusasco V, Burgos F, Casaburi R, Coates A, Crapo R, Enright P, van der Grinten CP, Gustafsson P, Jensen R, Johnson DC, MacIntyre N, McKay R, Navajas D, Pedersen OF, Pellegrino R, Viegi G, Wanger J, Force AET (2005) Standardisation of spirometry. Eur Respir J 26(2):319-338. doi:10.1183/09031936.05.00034805

21. Faria AC, Lopes AJ, Jansen JM, Melo PL (2009) Evaluating the forced oscillation technique in the detection of early smokinginduced respiratory changes. Biomed Eng Online 8:22. doi:10. 1186/1475-925X-8-22

22. Borsboom GJ, van Pelt W, van Houwelingen HC, van Vianen BG, Schouten JP, Quanjer PH (1999) Diurnal variation in lung function in subgroups from two Dutch populations: consequences for longitudinal analysis. Am J Respir Crit Care Med 159(4):1163-1171. doi:10.1164/ajrccm.159.4.9703106

23. Baruzzi A, Riva R, Cirignotta F, Zucconi M, Cappelli M, Lugaresi E (1991) Atrial natriuretic peptide and catecholamines in obstructive sleep apnea syndrome. Sleep 14(1):83-86

24. Horner RL, Brooks D, Kozar LF, Tse S, Phillipson EA (1995) Immediate effects of arousal from sleep on cardiac autonomic outflow in the absence of breathing in dogs. J Appl Physiol 79(1):151-162 
25. Somers VK, Dyken ME, Mark AL, Abboud FM (1993) Sympathetic-nerve activity during sleep in normal subjects. N Engl J Med 328(5):303-307. doi:10.1056/nejm199302043280502

26. Spaak J, Egri ZJ, Kubo T, Yu E, Ando S, Kaneko Y, Usui K, Bradley TD, Floras JS (2005) Muscle sympathetic nerve activity during wakefulness in heart failure patients with and without sleep apnea. Hypertension 46(6):1327-1332. doi:10.1161/01. HYP.0000193497.45200.66

27. Barnes PJ, Fitzgerald GA, Dollery CT (1982) Circadian variation in adrenergic responses in asthmatic subjects. Clin Sci 62(4):349-354

28. Kapsimalis F, Kryger MH (2002) Gender and obstructive sleep apnea syndrome, part 2: mechanisms. Sleep 25(5):499-506

29. Shore SA, Fredberg JJ (2005) Obesity, smooth muscle, and airway hyperresponsiveness. J Allergy Clin Immunol 115(5):925927. doi:10.1016/j.jaci.2005.01.064
30. Zeki AA, Franzi L, Last J, Kenyon NJ (2009) Simvastatin inhibits airway hyperreactivity: implications for the mevalonate pathway and beyond. Am J Respir Crit Care Med 180(8):731-740. doi:10. 1164/rccm.200901-00180C

31. Callaerts-Vegh Z, Evans KL, Dudekula N, Cuba D, Knoll BJ, Callaerts PF, Giles H, Shardonofsky FR, Bond RA (2004) Effects of acute and chronic administration of beta-adrenoceptor ligands on airway function in a murine model of asthma. Proc Natl Acad Sci USA 101(14):4948-4953. doi:10.1073/pnas.0400452101

32. Hetzel MR, Clark TJ (1980) Comparison of normal and asthmatic circadian rhythms in peak expiratory flow rate. Thorax 35(10):732-738

33. Joos GF, O'Connor B, Anderson SD, Chung F, Cockcroft DW, Dahlen B, DiMaria G, Foresi A, Hargreave FE, Holgate ST, Inman M, Lotvall J, Magnussen H, Polosa R, Postma DS, Riedler J (2003) Indirect airway challenges. Eur Respir J 21(6):1050-1068 